\title{
LOCALLY FREE ACTIONS AND STIEFEL-WHITNEY NUMBERS. II
}

\author{
R. E. STONG ${ }^{1}$ AND H. E. WINKELNKEMPER ${ }^{2}$
}

\begin{abstract}
This paper determines the possible bordism classes of manifolds with a locally free $G$ action for $G$ one of $S^{1} \times S^{1},\left(S^{1}\right)^{4}$ or $S^{3}$ and gets partial information for $\left(S^{1}\right)^{3}$.
\end{abstract}

1. Introduction. An action of a $k$-dimensional Lie group $G$ on an $n$-manifold $M^{n}$ is called locally free if all isotropy groups are discrete subgroups of $G$. In [4], Winkelnkemper analyzed the cobordism classes of smooth manifolds admitting locally free $S^{1} \times S^{1}$ actions. Unfortunately, at the bottom of page 328 of [4] there is the phrase "it follows easily", and as is to be anticipated the assertion is false.

The objective of this paper is to correct this error. For $S^{1} \times S^{1}$ the proper result is

THEOREM. The set of classes $\alpha \in \mathfrak{N}_{n}$ with $n \geqslant 2$ represented by a manifold $M^{n}$ admitting a locally free $S^{1} \times S^{1}$ action consists precisely of the classes with $w_{n}(\alpha)=0$.

Note. We restrict to $n \geqslant 2$ to avoid nonsense about $G$ actions on the empty manifold of dimension $n<2$.

We also determine the classes represented by manifolds with $\left(S^{1}\right)^{4}$ and $S^{3}$ actions and examine $\left(S^{1}\right)^{3}$ actions. Our Proposition 3.3 shows that the main point of $[4$, Proposition 2.4, p. 324] is correct. We merely illustrate with a different example.

2. Fiberings over projective spaces. In order to construct locally free $G$ actions, we will use certain standard fiberings over products of projective spaces.

For $\xi$ a vector bundle over a space $X$, let $R P(\xi)$ be the projective space bundle of $\xi$ consisting of lines in the fibers of $\xi$ and let $\lambda$ be the canonical line bundle over $R P(\xi)$ with total space the pairs $(\alpha, x)$, with $\alpha$ a line in a fiber of $\xi$ and $x$ a vector of $\xi$ in the line $\alpha$. Denote by $\underline{n}$ the trivial $n$-plane bundle

Received by the editors February 25, 1977.

AMS (MOS) subject classifications (1970). Primary 57D85.

${ }^{1}$ R. E. Stong is indebted to the National Science Foundation for financial support and to the Institute for Advanced Study for financial support and hospitality during his work.

${ }^{2}$ H. E. Winkelnkemper is indebted to the National Science Foundation for support under contract number MPS 74-07815-A01. 
over a space, and let $R P(n)=R P(n+1)$, where $n+1$ is the trivial bundle over a point, be projective $n$-space.

Let $R P\left(n_{1}, n_{2}, \ldots, n_{k}\right)$ be the projective space bundle of the bundle $\lambda_{1} \oplus \lambda_{2} \oplus \cdots \oplus \lambda_{k}$ over $R P\left(n_{1}\right) \times \cdots \times R P\left(n_{k}\right)$, where $\lambda_{i}$ is the pull-back of the canonical bundle over the $i$ th factor.

We also need a manifold which is slightly more complicated to describe. Over $R P(1) \times R P(1)$ one has a projective space bundle $R P\left(\lambda_{1} \oplus \underline{1}\right)$, with projection $\pi$ and canonical line bundle $\lambda$. Over $R P\left(\lambda_{1} \oplus \underline{1}\right)$ one has a projective space bundle $R P\left(\lambda \oplus \pi^{*} \lambda_{2} \oplus \underline{1}\right)$.

Proposition 2.1. There exist indecomposable elements $x_{n}, n \geqslant 4, n \neq 2^{t}-$ 1 , in $\mathfrak{R}_{n}$ which are represented by manifolds admitting a locally free $S^{1} \times S^{1}$ action.

Proof. If $n=4 s, s \geqslant 1$, let $x_{n}$ be the class of $R P(1, \ldots, 1,0) \quad$ (2s ones). If $n=4 s+2, s \geqslant 1$, let $x_{n}$ be the class of $R P(1, \ldots, 1,0,0,0) \quad(2 s$ ones). If $n=2^{p}(2 q+1)-1$, with $p, q>0$ and $2^{p} q=s+3 \geqslant 4$, let $x_{n}$ be the class of $\operatorname{RP}\left(2^{p}, 3,1, \ldots, 1,0\right) \quad\left(s\right.$ ones). Finally, if $n=5$, let $x_{n}$ be the class of the manifold $R P\left(\lambda \oplus \pi^{*} \lambda_{2} \oplus \underline{1}\right)$.

For $n \neq 5$, these classes $x_{n}$ are shown to be indecomposable in [2, Proposition 7.1], the same being noted in [3, page 187]. For $n=5$, indecomposability may be verified by direct calculation. One can, however, argue as follows. As noted in [3, page 187], $R P(2,1,0)$ is indecomposable and fibers over $R P(2) \times R P(1)$. The manifold $R P\left(\lambda_{1} \oplus \underline{1}\right)$ is $K \times R P(1)=R P(1,0) \times$ $R P(1)$, where $K$ is the Klein bottle, and under the map $f \times 1: R P(1,0) \times$ $R P(1) \rightarrow R P(2) \times R P(1)$ with $f$ pulling the canonical line bundle over $R P(2)$ back to that over $R P(1,0)$, the fibration of $R P(2,1,0)$ over $R P(2) \times R P(1)$ pulls back to $R P\left(\lambda \oplus \pi^{*} \lambda_{2} \oplus 1\right)$. Since $f \times 1$ is a degree one map, Proposition 2.4 of [2] applies, and the class of $R P\left(\lambda \oplus \pi^{*} \lambda_{2} \oplus \underline{1}\right)$ is decomposable.

It remains to exhibit $S^{1} \times S^{1}$ actions on our manifolds which are locally free. For $R P\left(n_{1}, \ldots, n_{k}\right)$, one notes that $R P\left(n_{1}, \ldots, n_{k}\right)$ is a quotient space of $S^{n_{1}} \times \cdots \times S^{n_{k}} \times S^{k-1}$ by an action of $Z_{2} \times \cdots \times Z_{2}(k+1$ times $)$. One has the obvious free action of $S^{1}$ on $S^{2 j+1}$, and by forming product actions, one obtains a locally free $S^{1} \times \cdots \times S^{1}$ ( $m$ times) action on $R P\left(n_{1}, \ldots, n_{k}\right)$ if $m \leqslant$ number of $n_{i}$ which are odd. Finally, $R P\left(\lambda \oplus \pi^{*} \lambda_{2} \oplus\right.$ 1) is the quotient space of $S^{1} \times S^{1} \times S^{1} \times S^{2}$ under the identifications induced by

$$
\begin{aligned}
\left(z_{1}, z_{2}, z_{3},(u, v, w)\right) & \sim\left(z_{1}, z_{2},-z_{3},(-u, v, w)\right), \\
& \sim\left(z_{1}, z_{2}, z_{3},(-u,-v,-w)\right), \\
& \sim\left(-z_{1}, z_{2},-\bar{z}_{3},(u, v, w)\right), \\
& \sim\left(z_{1},-z_{2}, z_{3},(u,-v, w)\right)
\end{aligned}
$$

where $z_{i} \in C,\left\|z_{i}\right\|=1,(u, v, w) \in \mathbf{R}^{3}$ with $u^{2}+v^{2}+w^{2}=1$ and the bar 
denotes complex conjugation. These identifications are compatible with the free action of $S^{1} \times S^{1}$ on $S^{1} \times S^{1} \times S^{1} \times S^{2}$ by

$$
\left(\left(z, z^{\prime}\right), \quad\left(z_{1}, z_{2}, z_{3},(u, v, w)\right)\right) \rightarrow\left(z \cdot z_{1}, z^{\prime} \cdot z_{2}, z_{3},(u, v, w)\right)
$$

inducing a locally free action of $S^{1} \times S^{1}$ on $R P\left(\lambda \oplus \pi^{*} \lambda_{2} \oplus \underline{1}\right)$.

Note. In the remark following Proposition 2.4 of [4] the manifold $M^{4}$ is $R P(1,1,0)$. This manifold does not bound, nor does $M^{6}=M^{4} \times R P(2)$. Thus, the remark in fact gives a counterexample to the proposition it is trying to illustrate. Recognition of this fact led to this paper.

As in [4], one has the observation that if a $k$-dimensional Lie group $G$ acts locally freely on a manifold $M^{n}$ then the action defines $k$ linearly independent vector fields on $M^{n}$ and hence for $i>n-k$ the Stiefel-Whitney classes $w_{i}$ vanish. Combining this with the proposition gives

THEOREM 2.2. The set of classes $\alpha \in \mathfrak{N}_{n}$ with $n \geqslant 2$ represented by $a$ manifold $M^{n}$ admitting a locally free $S^{1} \times S^{1}$ action consists precisely of the classes with $w_{n}(\alpha)=0$.

Proof. Both sets of classes are the elements of ideals in $\Re_{*}=Z_{2}\left[x_{i} \mid i \neq 2^{t}\right.$ - 1] described by $x_{2}=[R P(2)]$ and $w_{n}\left(x_{n}\right)=0$ if $n>2$. Since the classes represented by manifolds with locally free $S^{1} \times S^{1}$ action all have even Euler characteristic, one has an inclusion and the ideals must coincide.

\section{Higher dimensional groups.}

Proposition 3.1. The set of classes $\alpha \in \mathfrak{N}_{n}$ with $n \geqslant 4$ represented by a manifold $M^{n}$ admitting a locally free $\left(S^{1}\right)^{4}$ action consists precisely of the classes for which all Stiefel-Whitney numbers divisible by $w_{n}, w_{n-1}, w_{n-2}$ and $w_{n-3}$ are zero.

Proof. Using $\left(S^{1}\right)^{4}$ actions similar to those of $\$ 2$ on the manifolds used in [2, Proposition 7.2] gives the result.

Proposition 3.2. The set of classes $\alpha \in \mathfrak{N}_{n}$ with $n \geqslant 3$ represented by a manifold $M^{n}$ admitting a locally free $S^{3}$ action consists precisely of the classes for which all Stiefel-Whitney numbers divisible by $w_{n}, w_{n-1}$, and $w_{n-2}$ are zero.

Proof. If $i=4 k+2$, let $x_{i}$ be the class of $R P(3, \ldots, 3,0,0,0)(k$ threes). If $i=4 k \geqslant 8$, let $x_{i}$ be the class of $R P(3,3,0, \ldots, 0)(4 k-7$ zeros). If $i=2^{p}(2 q+1)-1, p, q>0$ and $2^{p} q=s+3 \geqslant 3$, let $x_{i}$ be the class of $R P\left(2^{p}, 3,1, \ldots, 1,0\right)$ ( $s$ ones). The criterion of [2, Lemma 3.4] shows that these are indecomposable in $\mathfrak{R}_{*}$, giving generators except in dimensions 2, 4 and 5 . Using quaternionic multiplication provides a locally free $S^{3}$ action on any $R P\left(3, n_{2}, \ldots, n_{k}\right)$.

By examining the characteristic number arguments in [2, Proposition 7.2] it suffices to show that the classes $x_{4}$ and $x_{5}$ may be chosen so that $x_{4}^{2}, x_{4} x_{5}$ and $x_{5}^{2}$ are represented by manifolds with locally free $S^{3}$ action, where $w_{4}\left(x_{4}\right)=0$.

To show that appropriate classes exist let: 
(a) $M^{8}=R P(3,3,0) \cup R P(\lambda \oplus 4)$ where $\lambda$ is the canonical line bundle over $R P(3,0)$,

(b) $M^{9}=R P(3,2,0,0,0) \cup R P(3,2,1,0)$, and

(c) $M^{10}=R P(3,1,1,0,0,0) \cup R P(3,5,0)$.

These manifolds are decomposable and have the characteristic numbers $s_{4,4}, s_{4,5}$ and $s_{5,5}$ nonzero. The relation between $s$-numbers and decomposability in $\mathfrak{R}_{*}$ completes the argument.

Note. If $M^{n}$ fibers over $R P(3)$, the tangent bundle of $M$ has 3 sections. These examples show that the set of classes in $\mathfrak{R}_{*}$ which fiber over $R P(3)$ is the same ideal.

Turning to actions of $\left(S^{1}\right)^{3}$ and making use of the analysis from [2, Proposition 7.2] as we did in Proposition 3.1, we see that the classes in $\mathfrak{R}_{*}$ represented by manifolds with a locally free $\left(S^{1}\right)^{3}$ action coincides with the set of classes described for $S^{3}$ actions if and only if there is an indecomposable $M^{6}$ with a locally free $\left(S^{1}\right)^{3}$ action.

In fact, there is no such manifold, or more precisely, we have

Proposition 3.3. If the closed manifold $M^{6}$ admits a locally free $\left(S^{1}\right)^{3}$ action, then $M^{6}$ bounds.

Note. $R P(3,0,0,0)$ is indecomposable and has three linearly independent vector fields as noted above.

Proof. Since $\left(S^{1}\right)^{3}$ is connected, it suffices to show that each component of $M$ bounds, and so we may assume $M$ connected. Without loss of generality, one may assume that the fixed set $F$ of $Z_{2} \times\{1\} \times\{1\}=Z_{2}$ on $M$ is a proper subset. (If $F$ is empty, $M$ bounds, and if $F=M,\left(S^{1}\right)^{3} / Z_{2} \cong\left(S^{1}\right)^{3}$ acts on $M$. Since $Z_{2^{s}} \times\{1\} \times\{1\}$ cannot act trivially for all $s$ in a locally free action, at some stage the fixed set cannot be all of $M$.)

Then $F$ consists of a disjoint union of submanifolds on each of which $\left(S^{1}\right)^{3}$ will act locally freely. Thus, $F=F^{5} \cup F^{4} \cup F^{3}$ and $M$ is bordant to $R P(\nu$ $\oplus \underline{1})$ where $\nu$ is the normal bundle of $F$ in $M$. This manifold $\operatorname{RP}(\nu \oplus \underline{1})$ is the union of the portions over the $F^{i}$, so it will suffice to see that each bounds.

In fact, more is true. $\mathfrak{R}_{6} \cong\left(Z_{2}\right)^{3}$ and is detected by the numbers associated to $w_{6}, w_{4} w_{2}$ and $w_{3}^{2}$. Since $M^{6}$ has $w_{6}$ and $w_{4} w_{2}$ zero, it will suffice to show that $w_{3}^{2}$ is zero on each portion of $R P(\nu \oplus \underline{1})$.

For $F^{5}, R P(\nu \oplus \underline{1})$ is a circle bundle over $F^{5}$, so bounds.

Each component of $F^{3}$ is the form $\left(S^{1}\right)^{3} / G_{x}$, where $G_{x}$ is an isotropy group, so is a copy of $\left(S^{1}\right)^{3}$. Looking at $R P(\nu \oplus \underline{1})=N$ where $\nu$ is a 3-plane bundle over $\left(S^{1}\right)^{3}$,

$$
w(N)=(1+c)^{4}+v_{1}(1+c)^{3}+v_{2}(1+c)^{2}+v_{3}(1+c)
$$

where $c=w_{1}(\lambda), v_{i}=w_{i}(\nu)$ and $c^{4}+v_{1} c^{3}+v_{2} c^{2}+v_{3} c=0$. Then $w_{3}(N)=$ $v_{1} c^{2}+v_{3}$ so $w_{3}(N)^{2}=0$ since $v_{1}^{2}=0$ and $v_{3}^{2}=0$ in $\left(S^{1}\right)^{3}$.

Now looking at a component of $F^{4}$, call it $P,\left(S^{1}\right)^{3}$ acts locally freely on $P$, so $\tau(P)=l \oplus \underline{3}$ where $\tau$ is the tangent bundle and $l$ is a line bundle. Letting $N=R P(\nu \oplus \underline{1})$ where $\nu$ is the normal bundle of $P$ in $M$, one has 


$$
w(N)=(1+\alpha)\left[(1+c)^{3}+v_{1}(1+c)^{2}+v_{2}(1+c)\right]
$$

where $\alpha=w_{1}(P), c=w_{1}(\lambda)$ and $v_{i}=w_{i}(\nu)$, giving

$$
w_{3}(N)=c^{2} \alpha+v_{2} \alpha
$$

since $c^{3}+v_{1} c^{2}+v_{2} c=0$. Then

$$
\begin{aligned}
w_{3}^{2}[N] & =\left(c^{4} \alpha^{2}+w_{2}^{2} \alpha^{2}\right)[N] \\
& =c^{4} \alpha^{2}[N]=c^{2}\left(v_{2}+v_{1}^{2}\right) \alpha^{2}[N] \\
& =\left(v_{2}+v_{1}^{2}\right) \alpha^{2}[P]=\left(v_{2}+v_{1}^{2}\right)^{2}[P],
\end{aligned}
$$

the latter equality following from the fact that $\alpha^{2}$ is the 2-dimensional $\mathrm{Wu}$ class in $P$.

From the results of Mostert [1] is is immediately clear that $P=Q^{3} \times S^{1}$, for some manifold $Q$. (To see this, note that by Mostert's analysis $P$, which has a locally free $\left(S^{1}\right)^{3}$ action can be constructed quite explicitly. The principal orbits are copies of $\left(S^{1}\right)^{3} / G_{x} \cong\left(S^{1}\right)^{3}=H$ and either $P=H \times$ $(P / H)=H \times\left(S^{1}\right)$ or $P$ is constructed using one or two copies of $Z_{2}$ inside $H$. Using the fact that $H$ has a unique copy of $\left(Z_{2}\right)^{3}$ inside it, the $Z_{2}$ 's can be taken as standard factors in $H$.) But in $Q^{3} \times S^{1}$ the squaring operation on $H^{2}\left(Q^{3} \times S^{1} ; Z_{2}\right)$ to $H^{4}\left(Q^{3} \times S^{1} ; Z_{2}\right)$ is trivial, and so

$$
w_{3}^{2}[N]=\left(v_{2}+v_{1}^{2}\right)^{2}[P]=0 .
$$

Thus, $M^{6}$ bounds.

Note. To our knowledge the analogous question of fibering an indecomposable $M^{6}$ over $\left(S^{1}\right)^{3}$, as raised in [2], is still unsettled.

\section{REFERENCES}

1. P. S. Mostert, On a compact Lie group acting on a manifold, Ann. of Math. 65 (1957), 447-455.

2. R. E. Stong, On fibering of cobordism classes, Trans. Amer. Math. Soc. 178 (1973), $431-447$.

3. _ Subbundles of the tangent bundle, Trans. Amer. Math. Soc. 200 (1974), 185-197.

4. H. E. Winkelnkemper, Locally free actions and Stiefel-Whitney numbers, Proc. Sympos. Pure Math., vol. 27, part I, Amer. Math Soc., Providence, R.I., pp. 323-330.

Department of Mathematics, University of Virginia, Charlottesville, Virginia 22903

Department of Mathematics, University of Maryland, College Park, Maryland 20742 